\title{
Application of Improved Deep Auto-Encoder Network in Rolling Bearing Fault Diagnosis
}

\author{
Jian Di, Leilei Wang \\ School of Control and Computer Engineering, North China Electric Power University, Baoding, China \\ Email: dijian6880@163.com;lywangleilei@163.com
}

How to cite this paper: $\mathrm{Di}, \mathrm{J}$. and Wang, L.L. (2018) Application of Improved Deep Auto-Encoder Network in Rolling Bearing Fault Diagnosis. Journal of Computer and Communications, 6, 41-53. https://doi.org/10.4236/jcc.2018.67005

Received: June 30, 2018

Accepted: July 27, 2018

Published: July 30, 2018

Copyright $\odot 2018$ by authors and Scientific Research Publishing Inc. This work is licensed under the Creative Commons Attribution International License (CC BY 4.0).

http://creativecommons.org/licenses/by/4.0/

\section{cc) (i) Open Access}

\begin{abstract}
Since the effectiveness of extracting fault features is not high under traditional bearing fault diagnosis method, a bearing fault diagnosis method based on Deep Auto-encoder Network (DAEN) optimized by Cloud Adaptive Particle Swarm Optimization (CAPSO) was proposed. On the basis of analyzing CAPSO and DAEN, the CAPSO-DAEN fault diagnosis model is built. The model uses the randomness and stability of CAPSO algorithm to optimize the connection weight of DAEN, to reduce the constraints on the weights and extract fault features adaptively. Finally, efficient and accurate fault diagnosis can be implemented with the Softmax classifier. The results of test show that the proposed method has higher diagnostic accuracy and more stable diagnosis results than those based on the DAEN, Support Vector Machine (SVM) and the Back Propagation algorithm (BP) under appropriate parameters.
\end{abstract}

\section{Keywords}

Fault Diagnosis, Rolling Bearing, Deep Auto-Encoder Network, CAPSO

Algorithm, Feature Extraction

\section{Introduction}

With the wide application of rolling bearings in various mechanical equipment, the problem of mechanical equipment damage caused by faults has become increasingly prominent, which affects the safety of equipment and personnel seriously [1]. Therefore, research on the fault diagnosis of rolling bearings is crucial for the stable operation of mechanical equipment. Vibration analysis is the most widely used method for detecting the health of mechanical equipment [2].

At present, there have been many studies on the diagnosis methods of bearing faults [3] [4] [5]. The analysis methods based on vibration signals mainly include noise method, oil sample analysis method, and vibration method. Hao et al. [6] 
proposed a multi-layer over-limit learning machine method to learn the fault vibration time domain signal and diagnose the fault of the rolling bearing. Liu et al. [7] proposed a method for extracting fault characteristics of rolling bearings based on fault characteristic trend line template. According to the fault characteristic trend line, the method finds the bearing fault characteristics and avoids the shortcomings in the order tracking process. A rolling bearing fault feature extraction method based on adaptive noise-based complete empirical mode decomposition (CEEMDAN) combined with IMF sample entropy is used to adaptively decompose the vibration signal [8]. In order to extract the fault characteristics of rolling bearings accurately and stably, Liu et al. [9] proposed a feature extraction method based on variational mode decomposition and singular value decomposition. However, the $\mathrm{K}$ value in this method needs to be given in advance, and the determination or range of other parameters is still lack of theoretical basis. The most critical part of the data-driven fault diagnosis method is the extraction of bearing fault characteristics [10]. Due to the increasing number of bearing equipment, the frequency of collecting samples is getting higher and higher, which makes bearing faults fall into massive data problems. The method adopted above requires a large amount of prior knowledge, rich theoretical knowledge of signal processing and practical experience as a support in the process of feature extraction. Moreover, the number of samples selected by the fault feature is small, which cannot adequately reflect the potential information of the bearing fault data, and reduces the accuracy of fault diagnosis. Therefore, it is especially important to choose a suitable method for fault diagnosis of rolling bearings.

In 2006, Professor Hinton proposed the idea of deep learning, which opened up a wave of deep learning in academia and industry [11]. At present, deep learning has also been applied in the field of mechanical failure. The advantage of this method lies in the ability to mine representative information and sensitive features from raw data. The convolutional neural network (CNN) is used to learn the characteristics from the frequency data of the vibration signal [12], and test the different performances of the feature learning from the original data, the spectrum and the time-frequency domain combined data. The method can adaptively learn features from frequency data and has higher diagnostic accuracy, but it is easy to fall into the local optimal solution. In order to combine the power transformer online monitoring of dissolved gas analysis (DGA) data in oil, Shi et al. [13] proposed a transformer fault diagnosis method based on CDAEN, and compared with the traditional method, the deep self-encoding algorithm can more vividly describe the potential of input data. Information and effective extraction of fault characteristics, but this method is poorly stable. Deep Auto-Encoder Network (DAEN) is a deep learning model and is widely used in various fields [14] [15]. In this paper, cloud adaptive particle swarm optimization (CAPSO) algorithm is used to optimize the depth self-encoding network, and CAPSO-DAEN based fault diagnosis method for rolling bearings is proposed. This method is based on the powerful computing of DAEN and combines 
the adaptive and stability of CAPSO algorithm search. It can optimize the connection weight of DAEN, reduce the constraint on the weight, and extract the fault characteristics automatically. The results show that the proposed method has higher accuracy and stable diagnosis results relatively.

\section{The Basic Theory of DAEN}

Deep Auto-Encoder Network (DAEN) is a neural network consisting of a multi-layer auto-encoder AE [16]. The output of the hidden layer of the above-mentioned $\mathrm{AE}$ is used as the input of the next level, layer by layer training. The feature representation in the original space is transformed into another feature space, and the potential information between the data is depicted, which can be applied to multi-layer network training and solve multi-classification problems. The training of the entire deep self-encoding network is divided into two stages: pre-training and fine-tuning [17].

\subsection{Pre-Training}

Auto-Encoder (AE) is a three-layer neural network model [18], which is composed of two parts: encoder and decoder. Its structure is shown in Figure 1.

Given the training samples $x=\left\{x_{1}, x_{2}, \ldots, x_{N}\right\}$, (for each sample $x_{i}$, $\left.x_{i}=\left[x_{1}, x_{2}, . ., x_{D}\right]^{T}\right)$. The output vector set of the hidden layer is $h=\left\{h_{1}, h_{2}, \ldots h_{N}\right\}$, (for each sample $h_{i}, h_{i}=\left[h_{1}, h_{2}, \ldots, h_{d}\right]^{T}$ ).

$$
\begin{aligned}
& h=s_{f}\left(W^{(1)} x+b^{(1)}\right) \\
& s_{f}(t)=1 /\left(1+e^{-t}\right)
\end{aligned}
$$

where $x$ and $h$ are D-dimensional vectors, $W^{(1)}$ is a weight matrix that connects the input layer to the hidden layer, $b^{(1)}$ is the offset matrix between the input layer and the hidden layer, $s_{f}$ is the activation function of the encoder. This paper uses the sigmoid activation function, which is used widely and has good

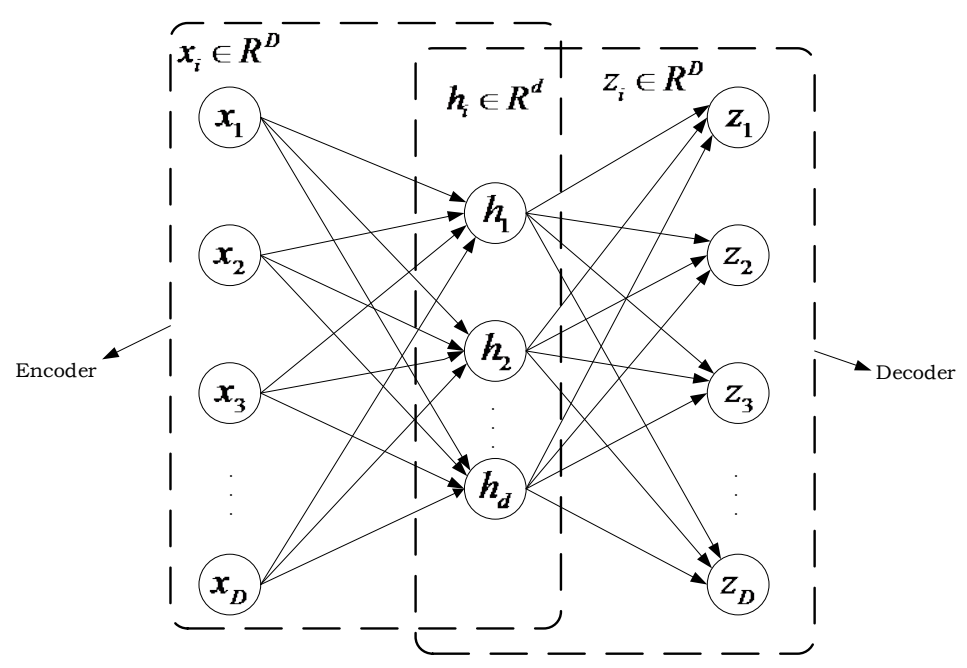

Figure 1. The structure of an autoencoder. 
experimental results. Where $t$ represents the input vector corresponding to each neuron [19].

The decoder is the inverse of the encoder, the original input vector is reconstructed using the hidden layer output vector obtained by the encoder. Output variable is $z=\left\{z_{1}, z_{2}, \ldots, z_{N}\right\}$, (for each sample $z_{i}, z_{i}=\left[z_{1}, z_{2}, \ldots, z_{D}\right]^{T}$ ) by decoders as follows

$$
z=s_{f}\left(W^{(2)} h+b^{(2)}\right)
$$

where $z$ is a $\mathrm{D}$-dimensional vector. $W^{(2)}$ is a weight matrix that connects the hidden layer to the output layer. $b^{(2)}$ is the offset matrix between the hidden layer and the input layer,

The goal of AE training is to optimal set of parameters $\theta=\left(W^{(1)}, b^{(1)}, W^{(2)}, b^{(1)}\right)$, so that the error between the output data and the input data is as small as possible [20]. AE usually uses MSE as the loss function of the standard autoencoder, the loss function expression is as follows

$$
J_{\mathrm{MSE}}(\theta)=\frac{1}{m} \sum_{i=1}^{m} L_{\mathrm{MSE}}\left(x_{i}, z_{i}\right)=\frac{1}{m} \sum_{i=1}^{m}\left(\frac{1}{2} P z_{i}-x_{i} P^{2}\right)
$$

\subsection{Fine-Tuning}

It can be known from the above pre-training process that when training the $\mathrm{AE}$ parameters of each layer, the other layer parameters are fixed and remain unchanged. Therefore, if you want better results, you can use the tagged data set to adjust all layer parameters of the entire depth self-encoding network to achieve global optimality by BP algorithm after the pre-training is completed. This process becomes fine-tuned. The fine-tuning after the pre-training is completed will directly use the BP algorithm to train the effect on the initial weight of the randomization, because the latter tends to fall into the local optimum [21].

\section{Bearing Fault Diagnosis Model Based on CAPSO-DAEN}

The input layer of the $\mathrm{AE}$ has the same number of nodes as the output layer. The process of encoding and decoding can be regarded as compressing the input data, and representing the high-dimensional original data with a low-dimensional vector, so that the compressed low-dimensional vector can be retained.

\subsection{CAPSO-DAEN Training Algorithm}

In this paper, the CAPSO algorithm is used to optimize the connection weight between each hidden layer and output layer in DAEN. It can find the optimal solution adaptively, reduce the dependence on the weight and improve the convergence speed of the algorithm. The input data for this model is the original time domain signal of the bearing vibration signal.

The hidden layer and the output layer of the $\mathrm{AE}$ are connected by weights, and the influence of the output vector of the hidden layer in the network on the output layer vector is different. The connection weight between each hidden 
layer and output layer in DAEN is mapped into the particles in the CAPSO algorithm, the speed and position of the particle are adjusted continuously, the fitness value is updated, and the global optimal solution is finally output. According to the expression of the loss function in Equation (4), the training error function $\mathrm{E}$ in the automatic encoder is the fitness function in the CAPSO algorithm, and the expression is as follows:

$$
E=\sum_{i=1}^{m}\left(\frac{1}{2} P z_{i}-x_{i} P^{2}\right)
$$

where $x_{i}$ is the $i$ th training sample of the AE encoder; $z_{i}$ is the $i$ th output vector of the $\mathrm{AE}$ encoder.

The specific steps for optimizing the weight between the hidden layer and the output layer in DAEN with CAPSO are as follows, as shown in Figure 2.

Step 1: Determining the network topology of the DAEN, determining the number of cells in each layer in the automatic encoder and initializing related parameters.

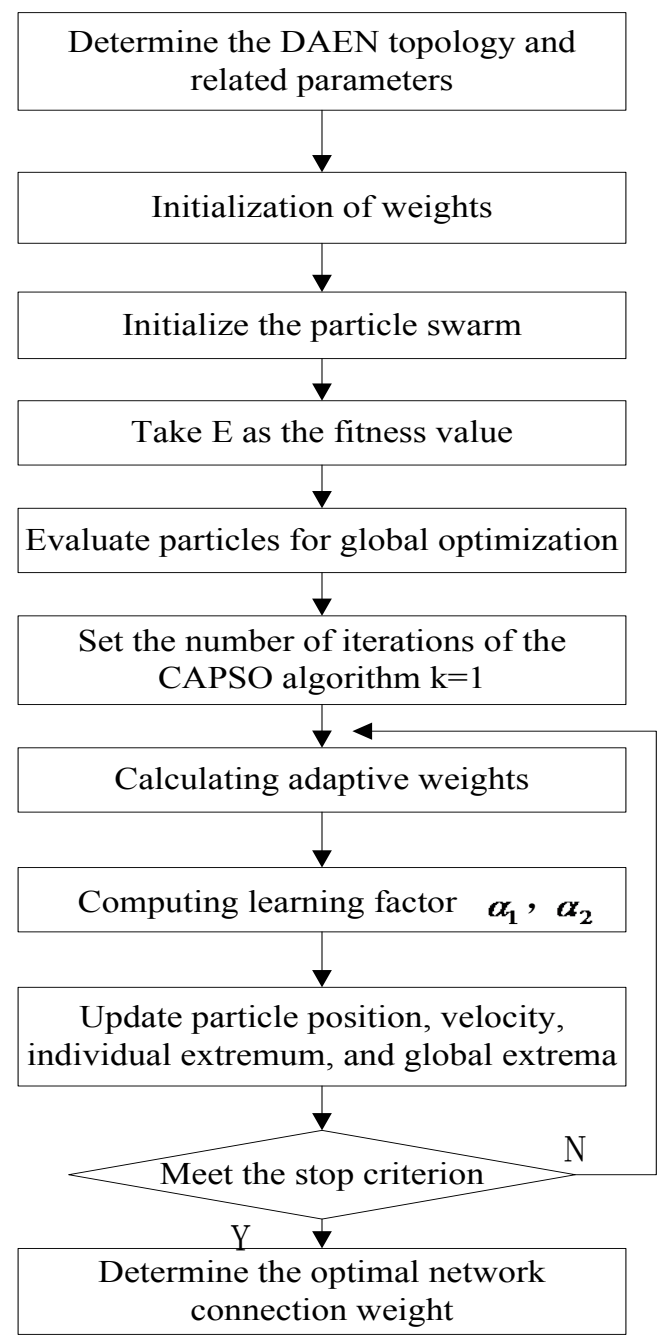

Figure 2. CAPSO-DAEN training algorithm flow chart. 
Step 2: Initialize the population in the CAPSO algorithm, Set the parameter vector $X=\{\omega, \theta\}$ of DAEN as the position $x_{i}^{k}$ of particle $i$ in the $k$ th iteration. Initialize the control coefficients $\alpha_{1}, \alpha_{2}$ in the CAPSO, the total number $\mathrm{N}$ of particle groups, the maximum number of iterations, and the initial value of the convergence angle $\gamma_{\min }$.

Step 3: The weights between the hidden layer and the output layer in the DAEN are mapped into particle groups in the CAPSO, the position and velocity of the particle group are initialized. Then, the initialized samples are input into the DAEN for adaptive training learning. According to the mode, the fitness function in the middle, constantly updating the fitness value $f$ of the particle iteration position.

Step 4: The particle individual optimal solution and the global optimal solution are updated to determine whether the end condition of the loop is satisfied. If the current number of iterations is the maximum number of iterations or the current error is the minimum error. The optimal network connection weight, is output. If the condition is not met, re-update the position and speed of the particle and execute Step3.

\subsection{Fault Diagnosis Model for Rolling Bearings}

The intelligent fault diagnosis model of rolling bearing based on CAPSO-DAEN is divided into the following steps, as shown in Figure 3, the steps are as follows:

Step 1: This model uses the original vibration time domain signal of the bearing as input data.

Step 2: The input data of the model is preprocessed. The vibration time domain

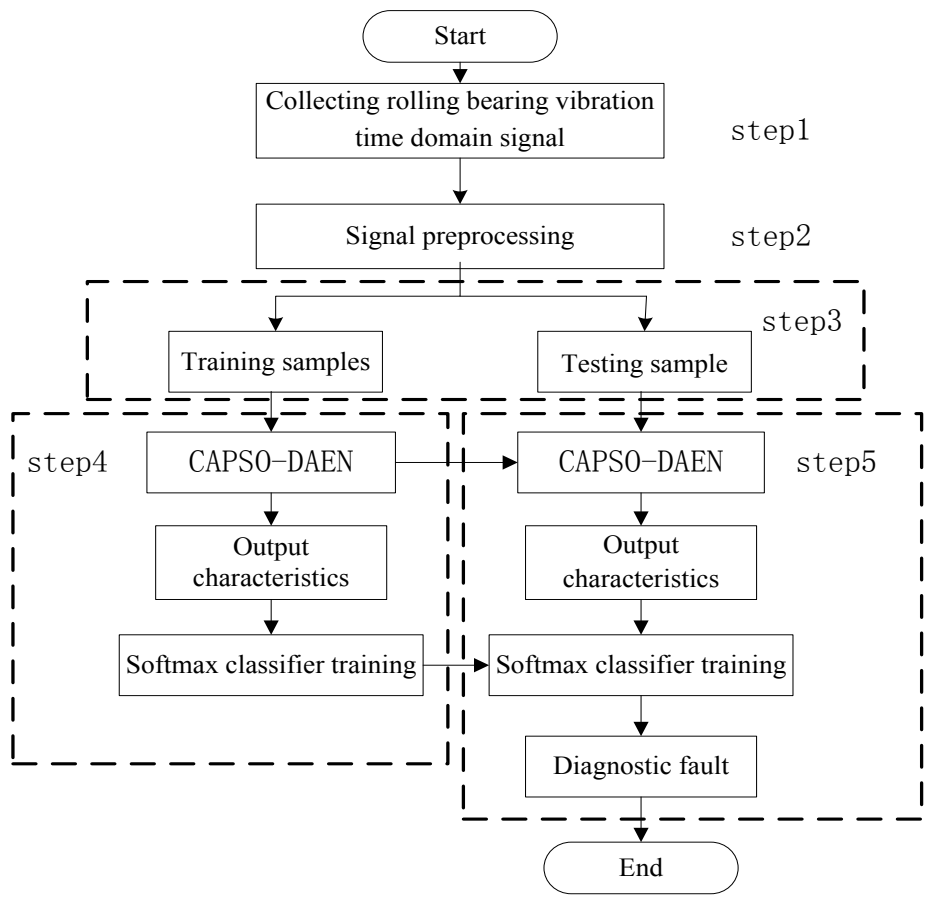

Figure 3. Bearing Fault Diagnosis Flow Chart Based on CAPSO-DAEN. 
signal is obtained by normalizing the original vibration time domain data. If the vibration time domain signal has a data length of $\mathrm{m}$, then there is

$$
\hat{x}_{i}=\frac{x_{i}-x_{\min }}{x_{\max }-x_{\min }}
$$

where $x_{i}$ is the th data point of the bearing vibration time domain signal $x_{1}$, $x_{\min }$ is the minimum data point of the bearing vibration time domain signal $x_{1}, x_{\min }$ is the maximum data point of the bearing vibration time domain signal $x_{1}, \hat{x}_{i}$ is the th data point of the vibration time domain signal $x_{2}, i=1,2$, $3, \ldots, \mathrm{m}$.

Step 3: The samples are divided into training samples and test samples. They are used to train and test CAPSO-DAEN respectively. Among them, the test samples are used to evaluate the effectiveness of CAPSO-DAEN extraction features, and test the accuracy of the model's fault diagnosis.

Step4: Aiming at the fault types of rolling bearings, the fault states of rolling bearings are coded. In the training sample stage. The CAPSO-DAEN training algorithm in Figure 3 is used to training and set some basic parameters used in CAPSO-DAEN. Because CAPSO-DAEN does not have the classification function, the Softmax classifier is used to train the output features. The training concentration label samples are used to fine tune the network and retain the training data for testing.

Step5: Sample testing stage. The CAPSO-DAEN trained in step 5 is used in the test work. The test samples are input into the trained CAPSO-DAEN to output the characteristics, and then the characteristics are input to the Softmax classifier. Lastly output the probability value of 7 faults. The state of the maximum probability value is the final fault diagnosis result, and then the classification results are judged. And evaluation of the accuracy of the model.

\section{Simulation and Analysis}

\subsection{Experimental Data}

Sample model of data should endeavor to include all bearing fault characteristics. Vibration signal contains complicated bearing information, thus time domain data of vibration signal are the inputs of network. The experimental data is based on the rolling bearing fault vibration data released by the Case Data Center of Case Western Reserve University. The CAPSO-DAEN method is used to learn the time domain signal of the rolling bearing vibration. In experiment, different degrees of single point damage were produced in the bearing outer ring, rolling body and inner ring by EDM technology.

In the experiment, the acceleration is used to collect the vibration signal, and the sensor is placed on the motor housing by using a magnetic base. The acceleration sensors are respectively placed at the drive end of the motor housing at 12 o'clock. The vibration signal was acquired by a 16-channel DAT recorder. The simulated experimental environment was MATLAB R2016a. The sampling frequency of the digital signal is $12,000 \mathrm{~Hz}$, and the drive end bearing fault data is 
simultaneously collected at a sampling rate of $48,000 \mathrm{~Hz}$.

In order to test the validity and accuracy of the algorithm, the sample set is divided into training samples and test samples, and 150 sample sets are randomly selected to test the CAPSO-DAEN algorithm, and the remaining sample set is used to train the CAPSO-DAEN algorithm.

\subsection{State Encoding}

The fault diagnosis method of rolling bearing based on CAPSO-DAEN is to solve the problem of multiple classification of faults. It is necessary to diagnose 7 kinds of fault problems of rolling bearings. The diagnosis results can be divided into 7 types, which are normal, outer ring one level fault, outer ring two level fault, rolling body one level fault, rolling body two level fault, inner ring one level fault and inner ring two level fault. As shown in Table 1, the fault states of rolling bearings are coded.

\subsection{Analysis of Result}

1) Research on fault diagnosis results of different AE layers

The average accuracy of fault diagnosis was tested when the number of $\mathrm{AE}$ layers was 0 - 15. The results are shown in Figure 4. As can be seen from Figure 4 , when the number of AE layers is 4, the average accuracy of fault diagnosis is already high, and then the correct rate increases slowly as the number of AE layers increases. In actual training, as the number of $\mathrm{AE}$ layers increases, the training time increases linearly. Taking into account the two factors of fault diagnosis and training time, the number of $\mathrm{AE}$ layers selected in the following test is 4 layers.

In order to further verify the extraction capability of the improved depth self coded network, two important components of the fourth layer features of the CAPSO-DAEN algorithm and the DAEN algorithm are analyzed, as shown in Figure 5.

2) Research on fault diagnosis results under different methods.

The fault diagnosis situation of training set is $300,500,700,900$, respectively. The results of CAPSO-DAEN and DAEN are shown in Table 2. Compared with

Table 1. Status coding of rolling bearings.

\begin{tabular}{cc}
\hline states of rolling bearing & coding \\
\hline normal & $(0,0,0,0,0,0,1)$ \\
outer ring one level fault & $(0,0,0,0,0,1,0)$ \\
outer ring two level fault & $(0,0,0,0,1,0,0)$ \\
rolling body one level fault & $(0,0,0,1,0,0,0)$ \\
rolling body two level fault & $(0,0,1,0,0,0,0)$ \\
inner ring one level fault & $(0,1,0,0,0,0,0)$ \\
inner ring two level fault & $(1,0,0,0,0,0,0)$ \\
\hline
\end{tabular}




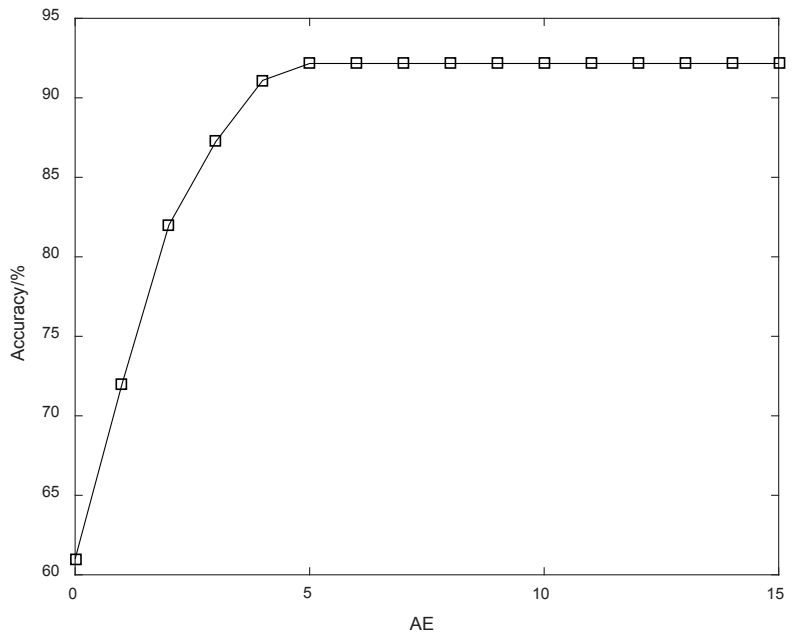

Figure 4. Fault diagnosis results when different AE layers.

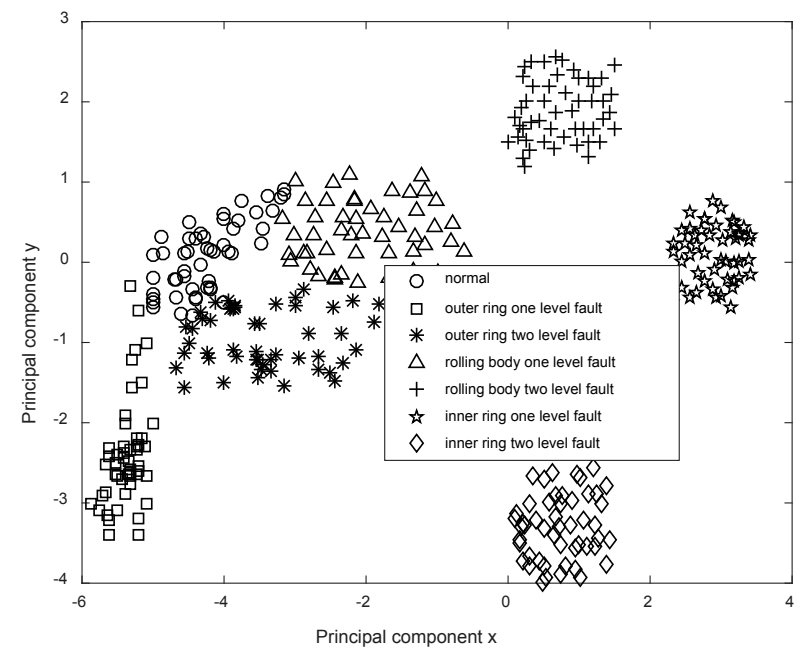

(a)

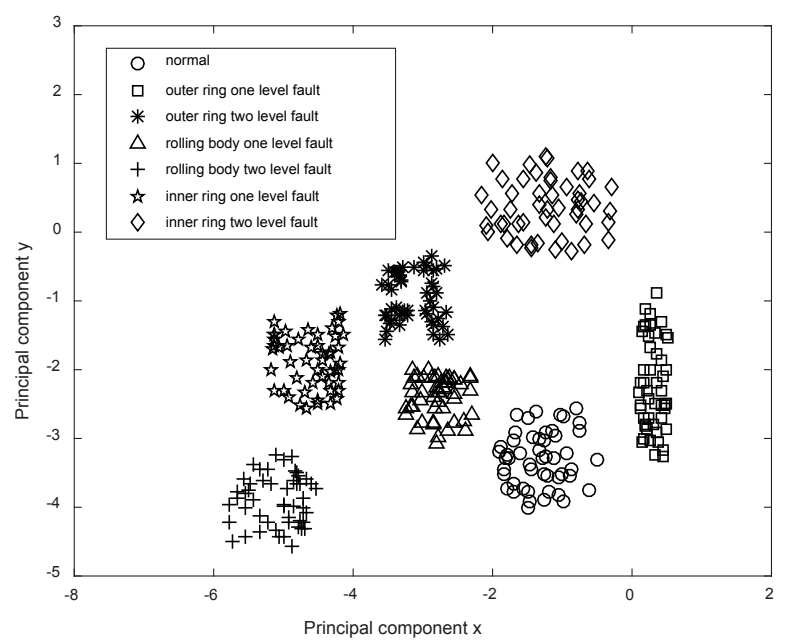

(b)

Figure 5. Characteristic scatter plot of two algorithms. (a) DAEN; (b) CAPSO-DAEN. 
Table 2. Fault diagnosis results for different training sets.

\begin{tabular}{ccccc}
\hline $\begin{array}{c}\text { Serial } \\
\text { number }\end{array}$ & training set & Test set & $\begin{array}{c}\text { CAPSO-DAEN average } \\
\text { diagnostic accuracy/\% }\end{array}$ & $\begin{array}{c}\text { DAEN average } \\
\text { diagnostic accuracy/\% }\end{array}$ \\
\hline 1 & 300 & 150 & 93.26 & 81.31 \\
2 & 500 & 150 & 94.47 & 83.76 \\
3 & 700 & 150 & 95.78 & 85.81 \\
4 & 900 & 150 & 96.01 & 87.23 \\
\hline
\end{tabular}

a few training samples, the more training samples, the better the diagnostic performance. Because more samples contain enough fault feature information, it can depict potential information between data, which is more conducive to extract fault characteristics. With the increasing number of training samples, the average accuracy of the two methods of CAPSO-DAEN and DAEN is also increasing. However, the average diagnostic accuracy of DAEN is significantly lower than that of CAPSO-DAEN, and the diagnostic accuracy of CAPSO-DAEN is higher. When the training set reaches 900, the average diagnostic accuracy of CAPSO-DAEN is $96.01 \%$, and the effect is better. Therefore, determining the number of training samples plays a key role in improving the accuracy of fault diagnosis.

In order to further verify the effectiveness of the CAPSO-DAEN algorithm, we compare it with traditional algorithms such as SVM and BP. The initial values of these parameters are determined through extensive experiments. SVM uses the most widely used radial basis kernel (RBF), in which the two most important parameters are the penalty factor and the radius of the kernel function, which are set to 20 and 0.082 respectively. The initial learning rate of BP algorithm is 0.1 , and the maximum training iteration number is 500 . The above 2 methods use the same vibration signal for training and testing, and the accuracy of the fault diagnosis is shown in Table 3.

From Table 2 and Table 3, we can see that the rolling bearing fault diagnosis method based on CAPSO-DAEN has higher fault diagnosis accuracy than the other 3 methods. In the test stage, the fault diagnosis method of rolling bearing based on CAPSO-DAEN and DAEN algorithm increases with the increase of training samples, but the accuracy rate of fault diagnosis is increasing, but CAPSO-DAEN has high accuracy. The fault diagnosis method of rolling bearing fault diagnosis based on BP and SVM algorithm increases with the increase of training samples and the accuracy rate of fault diagnosis. It is also increasing. However, when the training sample is 250 , the growth rate is slow, and it is only suitable for small sample training.

When the training sample set is 350 , the four methods of CAPSO-DAEN, DAEN, BP and SVM are tested, respectively, of which the number of AE layers in the CAPSO-DAEN method and the DAEN method is 4 , and the statistical diagnosis results are shown in Table 4. According to the table, the average diagnostic accuracy of the CAPSO-DAEN method was the highest, $94.46 \%$, higher 
Table 3. Fault diagnosis results of BP and SVM in different training sets.

\begin{tabular}{cccc}
\hline training set & Test set & $\begin{array}{c}\text { The BP method diagnoses } \\
\text { the average correct rate/\% }\end{array}$ & $\begin{array}{c}\text { The SVM method diagnoses } \\
\text { the average correct rate/\% }\end{array}$ \\
\hline 100 & 150 & 55.89 & 57.70 \\
150 & 150 & 60.51 & 62.11 \\
200 & 150 & 73.73 & 71.25 \\
250 & 150 & 78.14 & 79.32 \\
\hline
\end{tabular}

Table 4. Test results for 15 times.

\begin{tabular}{ccc}
\hline Method & Average diagnostic accuracy/\% & Standard deviation/\% \\
\hline CAPSO-DAEN & 94.46 & 0.79 \\
DAEN & 83.75 & 2.70 \\
BP & 78.83 & 3.94 \\
SVM & 79.94 & 2.15 \\
\hline
\end{tabular}

than the accuracy of the other 3 methods. In the standard deviation, the standard deviation of the CAPSO-DAEN method was significantly lower than the other two methods, $0.79 \%$, and the standard deviation of DAEN, BP and SVM were $2.70 \%, 3.94 \%, 2.15 \%$ respectively. Therefore, the CAPSO-DAEN method has a higher diagnostic accuracy than the DAEN method and the traditional BP and SVM methods, and the stability of the fault diagnosis is higher.

\section{Conclusions}

In this paper, an improved deep autoencoder network method is proposed for rotating machinery fault diagnosis. Firstly, the utility model can learn the vibration time domain signal of the rolling bearing directly, which can extract the fault feature automatically and effectively. Therefore, it overcome the problems existing in the traditional method in extracting the fault feature and does not need to manually extract the feature, thereby improving the effectiveness of the fault diagnosis. Secondly, the improved deep autoencoder network algorithm optimizes the connection weight by using the randomness and stability of the CAPSO algorithm search, reduces the constraint on the weight, and improves the learning efficiency of the algorithm.

The proposed method is applied to the fault diagnosis of rolling bearing. The results confirm that the proposed method has higher diagnostic accuracy and more stable diagnosis results than other methods. Besides, the proposed deep autoencoder network method has better classification performance than deep autoencoder network method.

\section{Conflicts of Interest}

The authors declare no conflicts of interest regarding the publication of this paper. 


\section{References}

[1] Henriquez, P., Alonso, J.B., Ferrer, M.A. and Travieso, C.M. (2014) Review of Automatic Fault Diagnosis Systems Using Audio and Vibration Signals. IEEE Transactions on Systems, Man, and Cybernetics. Systems, 44, 642-652. https://doi.org/10.1109/TSMCC.2013.2257752

[2] Lei, Y., He, Z. and Zi, Y. (2009) Application of an Intelligent Classification Method to Mechanical Fault Diagnosis. Expert Systems with Applications, 36, 9941-9948. https://doi.org/10.1016/j.eswa.2009.01.065

[3] Amar, M., Gondal, I. and Wilson, C. (2015) Vibration Spectrum Imaging: A Novel Bearing Fault Classification Approach. IEEE Transactions on Industrial Electronics, 62, 494-502. https://doi.org/10.1109/TIE.2014.2327555

[4] Grasso, M., Chatterton, S., Pennacchi, P. and Colosimo, B.M. (2016) A Data-Driven Method to Enhance Vibration Signal Decomposition for Rolling Bearing Fault Analysis. Mechanical Systems and Signal Processing, 81, 126-147. https://doi.org/10.1016/j.ymssp.2016.02.067

[5] Chen, K., Gu, S. and Zheng, H. (2018) Research on Real-Time Intelligent Fault Diagnosis Algorithm for Rolling Bearing. Journal of Electronic Measurement \& Instrumentation, 32, 66-72.

[6] Hao, L.N., Wang, F.L. and Cao, R.M. (2017) Multi-Layer Extreme Learning Machine Methods Based Fault Diagnosis of Rolling Bearing. Science Technology \& Engineering, 17, 86-91.

[7] Liu, D. (2017) Bearing Fault Diagnosis Based on Fault Characteristic Trend Template. Journal of Mechanical Engineering, 53, 083. https://doi.org/10.3901/JME.2017.09.083

[8] Zhao, L., Xia, J., Zehua, L.I., et al. (2017) Fault Diagnosis of Rolling Bearing Based on VMD Sample Entropy and LS-SVM. Journal of Military Transportation University, No. 3, 96-100.

[9] Liu, C., Wu, Y.J. and Zhen, C. (2015) Rolling Bearing Fault Diagnosis Based on Variational Mode Decomposition and Fuzzy C Means Clustering. Proceedings of the Csee, 35, 3358-3365.

[10] Thirukovalluru, R., Dixit, S., Sevakula, R.K., Verma, N.K. and Salour, A. (2016) Generating Feature Sets for Fault Diagnosis Using Denoising Stacked Auto-Encoder. Proceedings of IEEE Conference on Prognostics and Health Management, Ottawa, 20-22 June 2016, 1-7.

[11] Hinton, G.E. and Salakhutdinov, R.R. (2006) Reducing the Dimensionality of Data with Neural Networks. Science, 313, 504-507. https://doi.org/10.1126/science.1127647

[12] Jing, L., Zhao, M., Li, P., et al. (2017) A Convolutional Neural Network Based Feature Learning and Fault Diagnosis Method for the Condition Monitoring of Gearbox. Measurement, 111, 1-10. https://doi.org/10.1016/j.measurement.2017.07.017

[13] Xin, S., Zhu, Y., Ning, X., et al. (2016) Transformer Fault Diagnosis Based on Deep Auto-Encoder Network. Electric Power Automation Equipment, 36, 122-126.

[14] Su, S., Liu, Z., Xu, S., et al. (2015) Sparse Auto-Encoder Based Feature Learning for Human Body Detection in Depth Image. Signal Processing, 112, 43-52. https://doi.org/10.1016/j.sigpro.2014.11.003

[15] Huang, L., Wang, Y., Zhang, X., et al. (2017) Research on Noise Robustness of Speech Recognition Based on Deep Auto-Encoder Neural Network. Computer Engineering \& Applications, 53, 49-54. 
[16] Liu, K., Lin, J., Zheng, L., et al. (2018) Slow Moving Target Detection Based on Deep Self-Coding Network. Computer Engineering, 44, 129-134.

[17] Zabalza, J., Ren, J., Zheng, J., et al. (2016) Novel Segmented Stacked Autoencoder for Effective Dimensionality Reduction and Feature Extraction in Hyperspectral Imaging. Neurocomputing, 185, 1-10. https://doi.org/10.1016/j.neucom.2015.11.044

[18] Shao, H., Jiang, H., Zhao, H., et al. (2017) A Novel Deep Autoencoder Feature Learning Method for Rotating Machinery Fault Diagnosis. Mechanical Systems and Signal Processing, 95, 187-204.

[19] Dong, H.B., Dong-Jin, L.I. and Zhang, X.P. (2018) Particle Swarm Optimization Algorithm with Dynamically Adjusting Inertia Weight. Computer Science, 45, 98-102+139.

[20] Janssens, O., Slavkovikj, V., Vervisch, B., Stockman, K., Loccufier, M., Verstockt, S., Van de Walle, R. and Van Hoecke, S. (2016) Convolutional Neural Network Based Fault Detection for Rotating Machinery. Journal of Sound and Vibration, 377, 331-345. https://doi.org/10.1016/j.jsv.2016.05.027

[21] Li, C., Sánchez, R.V., Zurita, G., Cerrada, M. and Cabrera, D. (2016) Fault Diagnosis for Rotating Machinery Using Vibration Measurement Deep Statistical Feature Learning. Sensors, 16, 895. https://doi.org/10.3390/s16060895 